\title{
Effectiveness of the novel impression tray "cleftray" for infants with cleft lip and palate: a randomized controlled clinical trial
}

\author{
Ritesh Kalaskar ${ }^{1}$, Priyanka Bhaje ${ }^{1}$, Shruti Balasubramanian ${ }^{1}$, Ashita Kalaskar ${ }^{2}$ \\ ${ }^{1}$ Department of Pediatric and Preventive Dentistry, ${ }^{2}$ Department of Oral Medicine and Radiology, \\ Government Dental College \& Hospital, Nagpur, India
}

\begin{abstract}
J Korean Assoc Oral Maxillofac Surg 2021;47:82-90)
Objectives: Cleft lip and palate (CLP) is one of the most common congenital deformities with worldwide prevalence. It causes a range of issues for infants that mainly involve difficulty in feeding due to abnormal oronasal communication. For this purpose, feeding plates are provided to infants to act as an artificial palate to aid in feeding. The most crucial procedure in fabrication of a feeding plate is creation of the impression using the traditional finger technique or impression trays. This preliminary research aims to compare the effectiveness of novel impression trays with that of the traditional finger technique for recording impressions of infants with CLP.

Materials and Methods: This randomized controlled trial was conducted among 30 infants who were divided into two groups based on the method of obtaining impressions: Group I, finger technique; Group II, specialized acrylic tray (cleftray).

Results: Use of cleftray required less impression time, a reduced amount of material, no incidence of cyanosis/choking in infants, and lower anxiety among doctors compared to the traditional method. Additionally, there was no distortion of cleft impressions, recorded maxillary tuberosity, or other fine details. Therefore, the novel impression tray (cleftray) exhibited superior outcomes in all the parameters compared to the finger technique.

Conclusion: Within the limitations of this study, we conclude that impression trays are superior to the traditional finger, spoon, or ice cream stick methods of creating impressions of CLP. However, it is necessary to conduct more clinical trials on a larger population based on other parameters to compare the effectiveness of the two techniques to draw definitive conclusions.
\end{abstract}

Key words: Anxiety, Cleft lip, Cleft palate, Feeding methods, Dental impression technique

[paper submitted 2020. 9. 19 / revised 1st 2020. 11. 7, 2nd 2020. 12. 3 / accepted 2020. 12. 10]

\section{Introduction}

Cleft lip and palate (CLP) is one of the most common congenital deformities of the orofacial region, with a worldwide prevalence of 1.5 per 1,000 live births ${ }^{1,2}$. It affects nearly 35,000 infants every year in India, with a reported prevalence of $0.66 \%$ in the Nagpur region ${ }^{3}$. Among unilateral clefts, $70 \%$ are on the left side ${ }^{4}$.

Infants with CLP present numerous issues, with the fore-

\author{
Ritesh Kalaskar \\ Department of Pediatric and Preventive Dentistry, Government Dental \\ College \& Hospital, Hanuman Nagar, Medical Chowk, Nagpur 440003, \\ India \\ TEL: +91-8550938585 \\ E-mail: riteshpedo@gmail.com \\ ORCID: https://orcid.org/0000-0001-7403-3364
}

(a) This is an open-access article distributed under the terms of the Creative Commons Attribution Non-Commercial License (http://creativecommons.org/ licenses/by-nc/4.0/), which permits unrestricted non-commercial use, distribution, and reproduction in any medium, provided the original work is properly cited.

Copyright (C) 2021 The Korean Association of Oral and Maxillofacial Surgeons. All rights reserved. most being difficulty in feeding due to abnormal oronasal communication. This disrupted communication creates difficulty in sucking due to the inability to achieve a seal around the nipple of the mother's breast or the bottle. ${ }^{5}$. Additionally, it causes regurgitation of milk into the nasal cavity due to insufficient negative intraoral pressure ${ }^{6}$. This regurgitation could lead to life-threatening complications secondary to aspiration pneumonia, and the feeding difficulties hamper the physical and mental well-being of infants. CLP hampers the quality of life of infants as well as their caregivers? ${ }^{7}$. Therefore, timely intervention with a feeding plate is of the utmost priority for these infants.

A feeding obturator/plate is used as a prosthetic aid to obturate the cleft, restoring the separation between the nasal and oral cavities. The plate provides a stable platform against which the infant can press the nipple and extract milk ${ }^{8}$. The crucial step in fabrication of the feeding plate is the impression procedure, which is determined by patient positioning, technique, and impression material ${ }^{9}$. Traditionally, the fin- 
ger technique and stock trays have been used for recording impressions in with a desired quantity of loaded material. A cast was prepared on the impression, and a special tray was fabricated to be used for recording the secondary impression. However, these techniques present numerous disadvantages including aspiration of material, airway obstruction due to excessive material, distortion from difficulty in removal of the impression, and failure to record minute details and extend the CLP. Additionally, this technique is complex and involves prolonged chairside time ${ }^{10,11}$.

Therefore, we developed a novel impression tray (cleftray) for unilateral CLP infants with cleft on the left side. The tray is available in four sizes. The unique design of these trays allows for easy insertion and removal from the oral cavity without distortion of the recorded impression. It also overcomes the other aforementioned disadvantages of the finger technique. This study was planned to compare the effectiveness of novel impression trays with the traditional finger technique for recording impressions of CLP infants.

\section{Materials and Methods}

This randomized controlled trial was conducted from January 2016 to April 2019 in the Department of Pediatric and Preventive Dentistry, Government Dental College \& Hospital in collaboration with Visvesvaraya National Institute of Technology (VNIT), Nagpur, after obtaining approval from the Institutional Ethics Committee of Government Dental College \& Hospital (GDCHN/5730/2015 Dt. 23.09.2015). The written informed consent was obtained from all patients.

\section{Procedure for fabrication of the CLP impression tray (cleftray)}

Thirty positive replicas (casts) of one-week-old infants with Veau's Class III CLP (left side) were included in the study. Landmarks/points used for designing left-side CLP impression trays were marked on these positive replicas, and the distances between them were measured (millimeters) using digital Vernier calipers.(Table 1, Fig. 1) CAD/CAM software was used for designing the CLP impression trays. The mean values of the cleft measurements derived from the positive replicas were used for designing "size 0" left-side CLP impression trays. The tray was designed in such a way that its shape was similar to that of the maxillary jaw. A projection was incorporated in the tray design corresponding to the cleft on the left side (as per measured mean cleft depth) such that the right side of the impression tray was larger than the left side. This design allows the tray to come in close contact with the tissue of the cleft region, reducing the quantity of material required to create the impression. Additionally, flanges were incorporated into the tray borders to correspond to the depth of the sulcus (as per measured mean sulcus depth).(Fig. 2) Thereafter, impression tray sizes 1, 2, and 3 were designed 2 $\mathrm{mm}$ larger than the previous tray size in the anteroposterior and lateral dimensions.

Using the three-dimensional printing-fused deposition modeling (FDM) method, left-side CLP impression trays of sizes $0,1,2$, and 3 were fabricated.(Fig. 3. A) However, these fabricated trays were neither sturdy nor autoclavable. Therefore, for purposes of the study, specialized acrylic trays (cleftray) were fabricated using the denture fabrication proce-

Table 1. Landmarks used for designing left-side CLP impression trays

\begin{tabular}{|c|c|c|}
\hline Sr. No. & Landmarks & Description \\
\hline 1 & Anteroposterior length of the cleft (APLC) & $\begin{array}{l}\text { The distance from point } \mathrm{C} \text { to point } \mathrm{C} \text {. Point } \mathrm{C} \text { is an interception point of a line drawn } \\
\text { in the anteroposterior direction in the cleft region.(Fig. 1. A) }\end{array}$ \\
\hline 2 & $\begin{array}{l}\text { Length from alveolus to alveolus in the } \\
\text { anterior region (LAAAR) }\end{array}$ & $\begin{array}{l}\text { The distance between point } \mathrm{A} \text { and point } \mathrm{A} \text {. This is the minimum lateral distance on the } \\
\text { alveolar ridge in the anterior segment of the maxillary jaw.(Fig. 1. A) }\end{array}$ \\
\hline 3 & $\begin{array}{l}\text { Length from alveolus to alveolus in the } \\
\text { middle region (LAAMR) }\end{array}$ & $\begin{array}{l}\text { The distance from point } \mathrm{M} \text { to point } \mathrm{M} \text {. This is the maximum lateral distance on the } \\
\text { alveolar ridge in the middle segment of the maxillary jaw.(Fig. 1. A) }\end{array}$ \\
\hline 4 & $\begin{array}{l}\text { Length from alveolus to alveolus in the } \\
\text { posterior region (LAAPR) }\end{array}$ & $\begin{array}{l}\text { The distance from point } \mathrm{T} \text { to point } \mathrm{T} \text {. Point } \mathrm{T} \text { is the most posterior point of the } \\
\text { maxillary tuberosity.(Fig. 1. A) }\end{array}$ \\
\hline 5 & Sulcus depth in the anterior region (SDAR) & $\begin{array}{l}\text { The distance between the alveolar ridge and the base of the sulcus in the anterior } \\
\text { region of the maxillary jaw (Fig. 1. B) }\end{array}$ \\
\hline 6 & Sulcus depth in the middle region (SDMR) & $\begin{array}{l}\text { The distance between the alveolar ridge and the base of the sulcus in the middle region } \\
\text { of the maxillary jaw (Fig. 1. B) }\end{array}$ \\
\hline 7 & Sulcus depth in the posterior region (SDPR) & $\begin{array}{l}\text { The distance between the alveolar ridge and the base of the sulcus in the posterior } \\
\text { region of the maxillary jaw (Fig. 1. B) }\end{array}$ \\
\hline 8 & Cleft width $(\mathrm{CW})$ & The distance between the most anterior aspect of alveolar ridges (Fig. 1. B) \\
\hline
\end{tabular}

Ritesh Kalaskar et al: Effectiveness of the novel impression tray "cleftray" for infants with cleft lip and palate: a randomized controlled clinical trial. J Korean Assoc Oral Maxillofac Surg 2021 

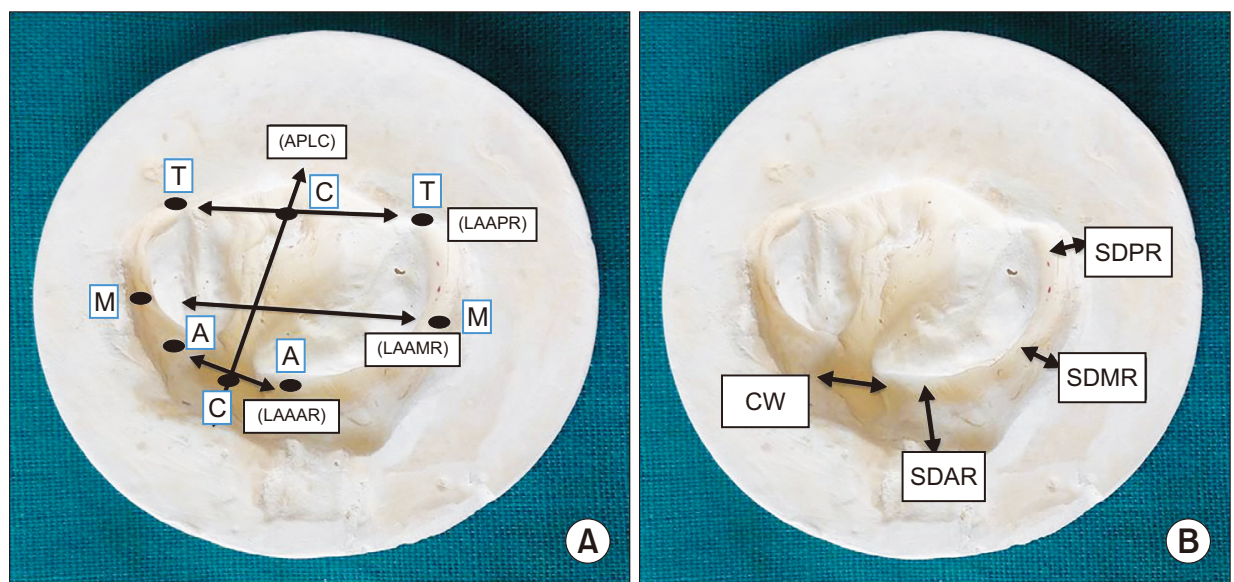

Fig. 1. Landmarks/points used for designing left-side cleft lip and palate impression trays. A. APLC (anteroposterior length of the cleft), LAAAR (length from alveolus to alveolus in the anterior region), LAAMR (length from alveolus to alveolus in the middle region), and LAAPR (length from alveolus to alveolus in the posterior region). B. SDAR (sulcus depth in the anterior region), SDMR (sulcus depth in the middle region), SDPR (sulcus depth in the posterior region), and CW (cleft width).

Ritesh Kalaskar et al: Effectiveness of the novel impression tray "cleftray" for infants with cleft lip and palate: a randomized controlled clinical trial. J Korean Assoc Oral Maxillofac Surg 2021

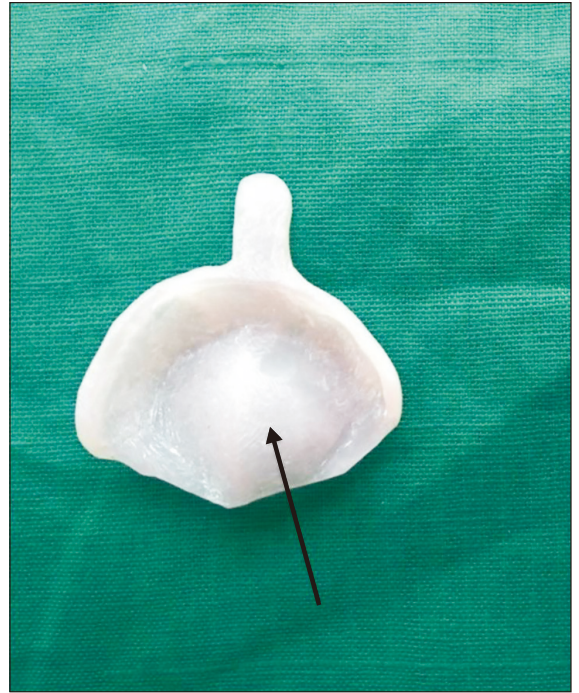

Fig. 2. Photograph of the cleft lip and palate impression tray showing the depression for the cleft.

Ritesh Kalaskar et al: Effectiveness of the novel impression tray "cleftray" for infants with cleft lip and palate: a randomized controlled clinical trial. J Korean Assoc Oral Maxillofac Surg 2021

dure (flasking, packing, and curing method).

Flasking of FDM impression trays (sizes 0, 1, 2, and 3): A mixture of Plaster of Paris (POP) was poured into the base of a flask, and the impression tray was placed over it and allowed to set (impression loading surface of the FDM impression tray was immersed in POP). A thin layer of cold Vaseline was applied over the set POP. The mid portion of the flask was placed over the initial set POP and filled with additional POP. After complete setting of the POP, the flask was separated into two sectional molds, and the FDM impression tray was removed. Thereafter, packing and curing of acrylic were performed similar to the denture fabrication procedure. After deflasking, a specialized acrylic impression tray was produced, finished, and polished, and its weight was recorded. (Fig. 3. B, Table 2)

For this study, acrylic trays were used to evaluate effectiveness since fabrication of stainless-steel trays was cost prohibitive.

A randomized controlled clinical trial was conducted on 60 infants (one week old) with non-syndromic Veau's Class III CLP (left side) who were reported to the department. The infants were divided into two groups of 30 each based on the technique of creating impressions of CLP.

In Group I (finger technique), impressions of CLP were created using the finger technique, whereas impressions in Group II (cleftray) were created using specialized acrylic impression trays. Computerized generated sequencing was used to allocate CLP infants to the groups.

\section{Impression formation (both groups)}

\section{1) Finger technique}

After securing the infant in the mother's/caretaker's lap, CLP impressions were taken using low-fusing impression compound (Y-DENTS impression compound). The index and middle fingers were loaded with the desired quantity of impression material and placed into the oral cavity by retracting the cheek and securing the airway. The material was adapted properly in the sulcular region and throughout the cleft region 

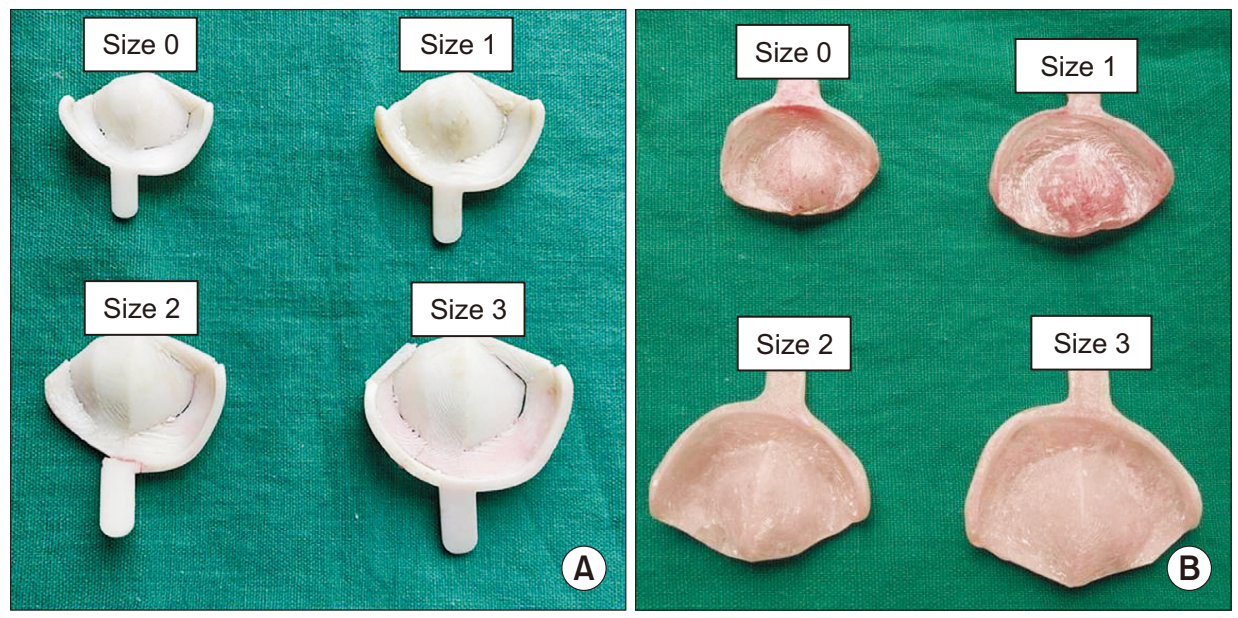

Fig. 3. Photographs of cleft lip and palate (CLP) impression trays. A. Fabricated using the fused deposition modeling method. B. Specialized acrylic CLP impression trays.

Ritesh Kalaskar et al: Effectiveness of the novel impression tray "cleftray" for infants with cleft lip and palate: a randomized controlled clinical trial. $J$ Korean Assoc Oral Maxillofac Surg 2021

Table 2. Weight of left-side cleft lip and palate impression trays

\begin{tabular}{ccc}
\hline Sr. No. & Size of the tray & Weight of the tray $(\mathrm{mg})$ \\
\hline 1 & 0 & 4.23 \\
2 & 1 & 5.43 \\
3 & 2 & 6.72 \\
4 & 3 & 8.20 \\
\hline
\end{tabular}

Ritesh Kalaskar et al: Effectiveness of the novel impression tray "cleftray" for infants with cleft lip and palate: a randomized controlled clinical trial. J Korean Assoc Oral Maxillofac Surg 2021

using the fingers. After ensuring that the material was set, it was carefully removed to avoid any distortion, and the oral cavity was assessed for any remaining material. The CLP impression (negative replica of the cleft) was cleaned under running water.

\section{2) Tray technique}

After securing the infant in the mother's/caretaker's lap, impression trays of the appropriate size were selected and verified to include the lateral maxillary segment, mucobuccal fold, and maxillary tuberosity.(Table 3) CLP impressions were taken using low-fusing impression compound. First, the impression tray was loaded with an exact amount of impression material and placed into the oral cavity after securing the airway. After approximately 1 minute of material setting, the impression tray was removed, and the oral cavity was assessed for any remaining material.

\section{Variables used for comparing Group I and Group ||}

Numerous variables were used to compare the effectiveness of the two groups. During CLP impression creation for both groups, time taken for the impression, heart rate of the oral health care provider (using a pulse oximeter), and presence of cyanosis/choking and crying of the infants were
Table 3. Size of specialized acrylic trays used in creating impressions of cleft lip and palate in Group 1

\begin{tabular}{cc}
\hline Size of the impression tray & Value \\
\hline 0 & $0(0)$ \\
1 & $27(90.0)$ \\
2 & $3(10.0)$ \\
3 & $0(0)$ \\
\hline
\end{tabular}

Values are presented as number (\%).

Ritesh Kalaskar et al: Effectiveness of the novel impression tray "cleftray" for infants with cleft lip and palate: a randomized controlled clinical trial. J Korean Assoc Oral Maxillofac Surg 2021

recorded. The same oral health care provider recorded the impression for all the infants in both groups. A pulse oximeter was placed on the middle finger (foot) of the oral health care provider so that they were not aware of their heart rate. After removal of the impressions from the oral cavity, any distortions in the impressions and the weights of the impressions were recorded (grams).(Fig. 4)

Thereafter, CLP impressions of both the groups were poured in dental stone, and positive replicas of the CLPs were recovered. On these positive replicas, transverse lateral dimension (LAAMR [length from alveolus to alveolus in the middle region]), anteroposterior dimension, and cleft width were measured (as mentioned in Fig. 1), and maxillary tuberosities were examined on the impressions.(Fig. 4) The feeding plates were fabricated and given to the infants of both groups. Use and maintenance of the feeding plate were explained to the parents per standard protocol.

\section{Statistical analysis}

Data were entered into a Microsoft Excel 2007 (Microsoft, Redmond, WA, USA) spreadsheet, and IBM SPSS Statistics (ver. 21.0; IBM, Armonk, NY, USA) was used for statistical 

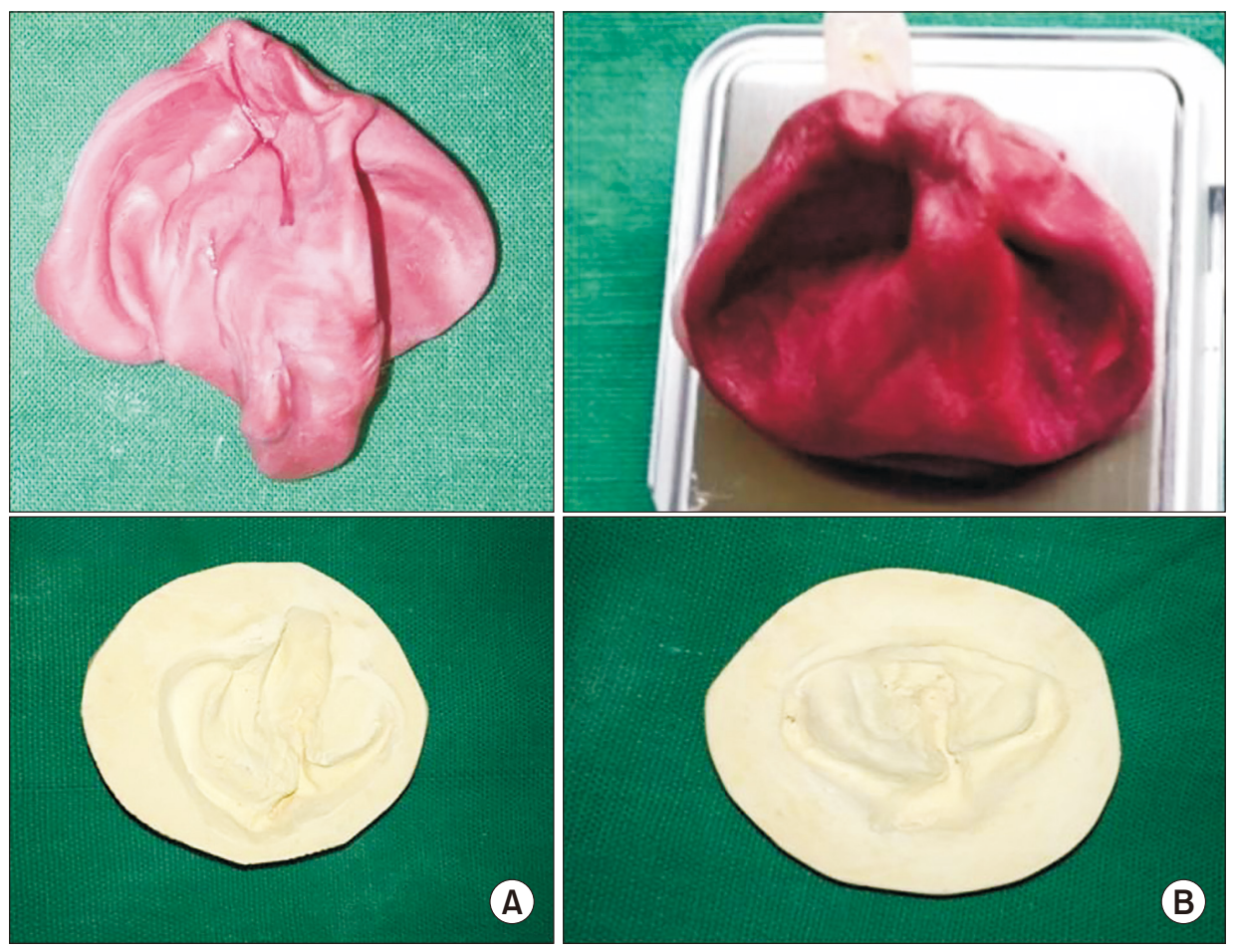

Fig. 4. Photographs of cleft lip and palate $(C L P)$ impressions and positive replicas of CLP (casts). A. Finger method. B. Specialized acrylic tray method. Ritesh Kalaskar et al: Effectiveness of the novel impression tray "cleftray" for infants with cleft lip and palate: a randomized controlled clinical trial. $J$ Korean Assoc Oral Maxillofac Surg 2021

Table 4. Mean heart rate of doctors, impression time, and amount of material required for cleft lip and palate impressions in both groups

\begin{tabular}{cccc}
\hline Parameter & Group I & Group II & $P$-value \\
\hline Heart rate (beats/min) & $93.3 \pm 11.1$ & $76.7 \pm 5.0$ & $<0.001$ \\
Impression time (min) & $12.6 \pm 3.4$ & $10.9 \pm 2.2$ & 0.02 \\
Amount of material & $16.4 \pm 2.8$ & $11.7 \pm 2.3$ & $<0.001$ \\
required $(\mathrm{g})$ & & & \\
\hline
\end{tabular}

Values are presented as mean \pm standard deviation.

Group I: finger technique, Group II: specialized acrylic tray (cleftray). Ritesh Kalaskar et al: Effectiveness of the novel impression tray "cleftray" for infants with cleft lip and palate: a randomized controlled clinical trial. J Korean Assoc Oral Maxillofac Surg 2021

analysis. Statistical significance was noted at $P \leq 0.05$. Independent $t$-test was used to compare the mean score of the variables.

\section{Results}

In $90 \%$ of the infants, a size 1 cleftray was used to create impressions of CLP.(Table 3) The mean heart rate of the oral health care provider during impression recording of CLP was 93.3 beats/min and 76.7 beats/min in Groups I and II, respectively, with a statistically significant difference $(P<0.001)$. The mean impression time for CLP with the tray method was 10.9 minutes, whereas the finger method required 12.6 minutes, a statistically significant difference $(P=0.02)$. The amount of impression compound needed to form impressions
Table 5. Influence of impression method on distortion of the impression, recording of maxillary tuberosity, cyanosis, and patient crying

\begin{tabular}{lcc}
\hline \multicolumn{1}{c}{ Parameter } & Group I & Group II \\
\hline $\begin{array}{l}\text { Distortion of the cleft lip and } \\
\text { palate impression }\end{array}$ & $17(56.7)$ & $0(0)$ \\
Maxillary tuberosity recording & $10(33.3)$ & $30(100)$ \\
Cyanosis/choking & $5(16.7)$ & $0(0)$ \\
Crying & $30(100)$ & $30(100)$ \\
\hline
\end{tabular}

Values are presented as number (\%).

Group I: finger technique, Group II: specialized acrylic tray (cleftray). Ritesh Kalaskar et al: Effectiveness of the novel impression tray "cleftray" for infants with cleft lip and palate: a randomized controlled clinical trial. J Korean Assoc Oral Maxillofac Surg 2021

in Groups I and II was $16.4 \mathrm{~g}$ and $11.7 \mathrm{~g}$, respectively, and the difference was statistically significant $(P<0.001)$. (Table 4$)$

Distortion of the CLP impression was observed in 56.7\% of the impressions created with the finger technique compared to the $0 \%$ of impressions made with the specialized acrylic tray technique. The tray method recorded $100 \%$ of the maxillary tuberosities, whereas the finger method showed only $33.3 \%$ of the maxillary tuberosities. This difference was statistically significant. Cyanosis or choking was observed in $16.7 \%$ of infants with the finger technique, whereas no such issues were observed using the tray technique. This difference was statistically significant.(Table 5)

The mean transverse dimension (on the positive replica) of LAAMR measured in Groups I and II were $39.4 \mathrm{~mm}$ and $38.3 \mathrm{~mm}$, respectively. This difference was not statistically 
Table 6. Mean transverse dimension, anteroposterior dimension, and cleft width recorded from positive replicas of cleft lip and palate from both groups

\begin{tabular}{lrrc}
\hline \multicolumn{1}{c}{ Parameter } & Group I & Group II & $P$-value \\
\hline $\begin{array}{l}\text { Mean transverse dimension } \\
(\mathrm{mm})\end{array}$ & $39.4 \pm 3.7$ & $38.3 \pm 3.7$ & 0.25 \\
Mean anteroposterior & & & \\
$\quad$ dimension & $29.5 \pm 3.4$ & $27.9 \pm 3.6$ & 0.08 \\
Cleft width in anterior region & $5.8 \pm 3.9$ & $6.2 \pm 3.5$ & 0.67 \\
Cleft width in middle region & $9.2 \pm 2.5$ & $9.8 \pm 2.6$ & 0.37 \\
Cleft width in posterior region & $10.1 \pm 3.2$ & $10.9 \pm 3.3$ & 0.34 \\
\hline
\end{tabular}

Values are presented as mean \pm standard deviation.

Group I: finger technique, Group II: specialized acrylic tray (cleftray). Ritesh Kalaskar et al: Effectiveness of the novel impression tray "cleftray" for infants with cleft lip and palate: a randomized controlled clinical trial. J Korean Assoc Oral Maxillofac Surg 2021

significant. The anteroposterior dimension of the CLP impression (on the positive replica) was 29.5 in Group I and 27.9 in Group II, which was not a significant difference. The cleft width in the anterior, middle, and posterior regions did not exhibit significant difference in either group.(Table 6)

\section{Discussion}

Infants born with CLP present with a range of issues that must be addressed by rehabilitation requiring multidisciplinary care from birth through adolescence. Primary care plays a vital role in these patients, who often have numerous healthcare needs including feeding difficulties, speech disorders, chronic ear infections, and orthodontic problems ${ }^{12}$. Among these, feeding difficulties remain the most problematic. Persistent oronasal communications due to the defect hinders proper suckling of milk and hampers speech, affecting the overall physical and mental growth of the infant ${ }^{13}$. Additionally, this communication causes regurgitation of milk, which can lead to aspiration pneumonia, posing a threat to life.

Due to inherent feeding difficulties, infants with CLP usually present with low birth weight and are malnourished. Therefore, they might not be able to withstand the stresses of corrective surgery immediately after birth. The pre-surgical period is used mainly to maintain adequate nutritional demand, promote weight gain, and prepare the child to combat infection by promoting healthy and comfortable feeding habits ${ }^{14}$.

Literature suggests approaches to resolve these feeding issues, including specially designed nipples with enlarged openings to promote ejection of milk with minimal effort and use of squeezable bottles instead of rigid feeding bottles ${ }^{15,16}$. However, these options are inadequate for large clefts. Use of nasogastric and orogastric tubes is effective, but they can be used only for a limited period of time ${ }^{17}$. Therefore, keeping in mind the aforementioned problems, timely intervention with feeding plate/obturator is necessary.

The concept of early treatment of CLP infants with feeding obturators was pioneered by $\mathrm{McNeil}^{18}$. Feeding plates/ obturators are passive plates that provide an artificial palate and closes the oronasal communication. The plate facilitates feeding, reduces nasal regurgitation, prevents choking, and shortens feeding time ${ }^{19}$. It also helps in positioning the tongue correctly to enable its functional role in development of the jaws and contributes to speech development. The obturator reduces passage of food into the nasopharynx, decreasing the incidence of otitis media and nasopharyngeal infections ${ }^{20}$. It also has a positive impact on the physical and psychological development of the infant and fosters the mother-child bond. This aids in establishing a sense of security and enhances the mental ability of the child, improving their overall quality of life at an early stage. Therefore, various specialists posit that pre-surgical management should be undertaken as soon as possible after birth.

The most crucial step in fabrication of a feeding plate is creation of the impression. Impression procedures in cleft infants pose a unique set of challenges that includes size constraints of the oral cavity, anatomical variations, lack of ability of the infant to cooperate and respond to commands, cyanotic episodes, poor nasopharyngeal reflexes, obligatory nasal breathing difficulties in adaptation of stock instruments, and difficulty in retrieval of the impressions. Therefore, while preparing an impression, proper patient and dentist positioning is an essential prerequisite for a safe and accurate procedure $^{21}$. Specialists use various techniques for making impressions, including the finger technique, use of spoon or ice cream sticks, and use of trays fabricated from pre-existing casts of other patients. The traditional methods of taking impressions have several disadvantages with respect to patient safety and technicality of the procedure. These include risk of aspiration of material, airway obstruction, and distortion from difficulty in removal of the impression, failure to record minute details and extension of the cleft region, and need for a secondary impression. However, with a cleftray, it is possible to use the exact amount of impression material to prevent the risk of aspiration and choking, reducing patient discomfort and material waste. It was observed in this study that the amount of impression compound required for the tray method was significantly smaller, and that $0 \%$ distortion was observed, leading to properly recorded details.

The impression technique used should properly record the 
lateral maxillary segments, maxillary tuberosities, and enable good reproduction of the mucobuccal folds ${ }^{7}$. For the present study, we created novel impression trays (cleftray) for leftsided unilateral CLP infants. Based on the mean values of the measurements, a tray size 0 was designed. These measurements were of crucial landmarks of cleft-like cleft width, anteroposterior dimension of clefts, alveolar dimensions, and sulcus depth to increase the accuracy of the impression due to the standardized dimensions. However, the size 0 tray might not be suitable for every infant because of different jaw sizes. Therefore, for this purpose, other trays were prepared through subsequent addition of material $2 \mathrm{~mm}$ antero-posteriorly and laterally to the mean dimensions. Availability of trays in four sizes renders it universally applicable for Veau's Class III (left side) infants of different jaw sizes, as with metal stock trays. Additionally, larger trays would be useful for taking impressions for feeding plates or nasoalveolar molding at later ages as jaw size increases, adding to its applicability for infants up to 6 months.

In this study, size 1 trays fit $90 \%$ of the infants and allowed accurate record of the details of clefts. When recording impressions of CLP, it is crucial to record the transverse details for obtaining good fit of feeding plates. The impressions recorded with impression trays did not differ in terms of transverse or anteroposterior dimensions compared to the finger technique. Another important anatomical structure is the maxillary tuberosity, which is essential for plate retention. These structures were recorded in $100 \%$ of the impressions formed using the tray method and in $33.3 \%$ of impressions using the finger technique.

Additionally, impression materials influence the safety and accuracy of the procedure. In this study, we used impression compound since it can be easily removed in cases of an emergency and exhibits better tear resistance compared to other impression materials.

The traditional procedure of taking impressions for CLP instills a great level of anxiety among oral health providers, which can lead to several complications including difficulty in removal of impressions, cyanotic events, and asphyxiation $^{22,23}$. Anxiety as measured by heart rate was significantly lower when the oral health care provider used the tray method compared to the finger technique, indicating greater control when using the tray technique. The utmost priority of the dentist when recording impressions is the safety of the infant. Jacobson and Rosenstein ${ }^{24}$, Brecht et al. ${ }^{25}$, and Kamble et al. ${ }^{26}$ highlighted the importance of safeguarding the respiratory system against complications such as cyanosis and mechanical obstruction from the impression material. In this study, cyanosis was observed in $16.7 \%$ of infants with the finger technique and none with the tray technique. Yilmaz et al. ${ }^{27}$ stated that oxygen saturation $\left(\mathrm{SpO}_{2}\right)$ monitoring should be the main focus during the impression process. A notable change in $\mathrm{SpO}_{2}$ value (5\% and greater) at any stage during the impression procedure requires safeguard protocols such as continuous supplemental oxygen delivery to prevent permanent damage to vital organs.

A study conducted by Dalessandri et al. ${ }^{28}$ compared an intraoral scanner (IOS) with the tray and putty method for creating impressions of CLP. They reported that parents considered IOS to be less invasive and clinicians were less stressed with its use. IOSs recorded better details and did not exhibit adverse events. They concluded that digital protocols accelerate the processing of molding plates with higher accuracy. Unfortunately, these advanced techniques are not available at all centers treating CLP infants especially in remote areas. However, cleftray could be readily available and is easy to use without any investment, adding to its applicability. An innovation of individualized CLP trays was put forth by Strobel-Schwarthoff et al. ${ }^{29}$. They devised trays made of cobaltchromium-molybdenum alloys that were prepared using wax patterns created on casts poured in polymethylmethacrylate trays. In this study, the acrylic trays were prepared from FDM trays based on exact CLP measurements of infants, adding to the accuracy of trays used in this study.

Through this preliminary research, it is evident that use of impression trays not only provides fine details of the cleft region, but also is more comfortable for infants and poses no threat to their life. Due to the ease of use and ability to obtain more control over the procedure, use of trays does not instill anxiety among oral health professionals when dealing with such infants. The trays used in this research were acrylic, taking into account the high cost of stainless-steel trays. However, future trays will be prepared using stainless steel manufactured in bulk to provide better application. Since the tray dimensions are based on measured parameters, ideal fit can be obtained despite being produced in bulk like stock trays while also being autoclavable and sturdy. The cleftrays used in this study were designed exclusively for Veau's Class III (left side) patients. It is recommended that similar trays be created for the right side as well as for Veau's class IV CLP patients. Additionally, similar studies in other populations should be performed to add credibility to the effectiveness of cleftray. 


\section{Conclusion}

This preliminary research shows the superiority of novel cleftray over the traditional finger technique in terms of recording details of CLP and infant safety. It is necessary to conduct clinical trials on other populations with larger sample sizes to further evaluate the effectiveness of cleftray.

\section{ORCID}

Ritesh Kalaskar, https://orcid.org/0000-0001-7403-3364

Priyanka Bhaje, https://orcid.org/0000-0003-1464-5478

Shruti Balasubramanian, https://orcid.org/0000-0002-76663981

Ashita Kalaskar, https://orcid.org/0000-0002-1415-5033

\section{Authors' Contributions}

R.K. conceived the idea and participated in data collection. P.B. performed the clinical procedure. S.B. participated in data interpretation and manuscript drafting. A.K. edited the manuscript. All authors read and approved the final manuscript.

\section{Acknowledgements}

We would like to thank Dr. Rashmi Uddhanwadiker, Associate Professor, Visvesvaraya National Institute of Technology of Nagpur, for supporting us in developing "cleftray".

\section{Ethics Approval and Consent to Participate}

The study was approved by the Institutional Ethics Committee of Government Dental College \& Hospital (GDCHN/5730/2015 Dt. 23.09.2015), and the written informed consent was obtained from all patients.

\section{Conflict of Interest}

No potential conflict of interest relevant to this article was reported.

\section{References}

1. Grayson BH, Garfinkle JS. Early cleft management: the case for nasoalveolar molding. Am J Orthod Dentofacial Orthop 2014;145:134-42. https://doi.org/10.1016/j.ajodo.2013.11.011
2. Mossey PA, Shaw WC, Munger RG, Murray JC, Murthy J, Little J. Global oral health inequalities: challenges in the prevention and management of orofacial clefts and potential solutions. Adv Dent Res 2011;23:247-58. https://doi.org/10.1177/0022034511402083

3. Kalaskar R, Kalaskar A, Naqvi FS, Tawani GS, Walke DR. Prevalence and evaluation of environmental risk factors associated with cleft lip and palate in a central Indian population. Pediatr Dent 2013;35:279-83

4. Rangeeth BN, Ahmed S, Cholan R, Russia M, Mohammed Raffi AJ. Role of the pediatric dentist and prosthodontist in early cleft management: presentation of two case reports. SRM J Res Dent Sci 2013;4:173-6. https://doi.org/10.4103/0976-433X.125600

5. Lodaya R, Dave A, Kunte S, Shah R. A feeding appliance for a 2 day old neonate with cleft lip and palate: a case report. Int J Oral Health Med Res 2017;3:86-9.

6. Ravichandra KS, Vijayaprasad KE, Vasa AA, Suzan S. A new technique of impression making for an obturator in cleft lip and palate patient. J Indian Soc Pedod Prev Dent 2010;28:311-4. https://doi. org/10.4103/0970-4388.76165

7. Emeka CI, Adeyemo WL, Ladeinde AL, Butali A. A comparative study of quality of life of families with children born with cleft lip and/or palate before and after surgical treatment. J Korean Assoc Oral Maxillofac Surg 2017;43:247-55. https://doi.org/10.5125/jkaoms.2017.43.4.247

8. Agarwal A, Rana V, Shafi S. A feeding appliance for a newborn baby with cleft lip and palate. Natl J Maxillofac Surg 2010;1:91-3. https://doi.org/10.4103/0975-5950.69149

9. Rizwaan AS, Sujoy B, Rajlakshmi B, Atif K. Prosthetic rehabilitation of cleft compromised newborns: a review. J Clin Diagn Res 2010;4:3632-8.

10. Lipp MJ, Lubit EC. An impression procedure for the neonatal patient with a cleft palate. Spec Care Dentist 1988;8:224-7. https:// doi.org/10.1111/j.1754-4505.1988.tb00741.x

11. Xepapadeas AB, Weise C, Frank K, Spintzyk S, Poets CF, Wiechers $\mathrm{C}$, et al. Technical note on introducing a digital workflow for newborns with craniofacial anomalies based on intraoral scans part I: 3D printed and milled palatal stimulation plate for trisomy 21. BMC Oral Health 2020;20:20. https://doi.org/10.1186/s12903020-1001-4

12. Taib BG, Taib AG, Swift AC, van Eeden S. Cleft lip and palate: diagnosis and management. Br J Hosp Med (Lond) 2015;76:584-5, 588-91. https://doi.org/10.12968/hmed.2015.76.10.584

13. Proffit WR, Fields HW. Contemporary orthodontics. 3rd ed. St. Louis: Mosby; 2000:287-8.

14. Jones JE, Henderson L, Avery DR. Use of a feeding obturator for infants with severe cleft lip and palate. Spec Care Dentist 1982;2:116-20. https://doi.org/10.1111/j.1754-4505.1982.tb01297. $\mathrm{x}$

15. Brine EA, Rickard KA, Brady MS, Liechty EA, Manatunga A, Sadove M, et al. Effectiveness of two feeding methods in improving energy intake and growth of infants with cleft palate: a randomized study. J Am Diet Assoc 1994;94:732-8. https://doi. org/10.1016/0002-8223(94)91938-0

16. Shaw WC, Bannister RP, Roberts CT. Assisted feeding is more reliable for infants with clefts--a randomized trial. Cleft Palate Craniofac J 1999;36:262-8. https://doi.org/10.1597/15451569_1999_036_0262_afimrf_2.3.co_2

17. Saunders ID, Geary L, Fleming P, Gregg TA. A simplified feeding appliance for the infant with a cleft lip and palate. Quintessence Int 1989;20:907-10.

18. McNeil CK. Congenital oral deformities. Br Dent J 1956;101:1916.

19. Goldberg WB, Ferguson FS, Miles RJ. Successful use of a feeding obturator for an infant with a cleft palate. Spec Care Dentist 1988;8:86-9. https://doi.org/10.1111/j.1754-4505.1988.tb00699.x

20. Oliver HT. Construction of orthodontic appliances for the treatment of newborn infants with clefts of the lip and palate. Am J Orthod 
1969;56:468-73. https://doi.org/10.1016/0002-9416(69)90208-5

21. Sabarinath VP, Hazarey PV, Ramakrishna Y, Vasanth R, Girish K. Caring for cleft lip and palate infants: impression procedures and appliances in use. J Indian Prosthodont Soc 2009;9:76-80. https:// doi.org/10.4103/0972-4052.55248

22. Rathee M. Single visit feeding appliance for 1-day-old neonate with cleft palate using safe dental putty-gauze hybrid impression technique for maxillary impression. J Surg Tech Case Rep 2015;7:7-11. https://doi.org/10.4103/2006-8808.184938

23. Chate RA. A report on the hazards encountered when taking neonatal cleft palate impressions (1983-1992). Br J Orthod 1995;22:299307. https://doi.org/10.1179/bjo.22.4.299

24. Jacobson BN, Rosenstein SW. Early maxillary orthopedics for the newborn cleft lip and palate patient. An impression and an appliance. Angle Orthod 1984;54:247-63. https://doi.org/10.1043/00033219(1984)054<0247:EMOFTN $>2.0$. CO;2

25. Brecht LE, Grayson BH, Cutting CB. Nasoalveolar molding in early management of cleft lip and palate. In: Taylor TD, ed. Clinical maxillofacial prosthetics. Chicago: Quintessence; 2000:63-84.

26. Kamble VD, Parkhedkar RD, Sarin SP, Patil PG, Kothari B. Simplifying cleft surgery by presurgical nasoalveolar molding (PNAM) for infant born with unilateral cleft lip, alveolus, and palate: a clinical report. J Prosthodont Res 2013;57:224-31. https://doi. org/10.1016/j.jpor.2013.03.002
27. Yılmaz RBN, Çakan DG, Noyan A. Comparison of oxygen saturation during impression taking before and after presurgical orthopedic therapy in babies with cleft lip and palate. Cleft Palate Craniofac J 2017;54:582-7. https://doi.org/10.1597/15-132

28. Dalessandri D, Tonni I, Laffranchi L, Migliorati M, Isola G, Bonetti $\mathrm{S}$, et al. Evaluation of a digital protocol for pre-surgical orthopedic treatment of cleft lip and palate in newborn patients: a pilot study. Dent J (Basel) 2019;7:111. https://doi.org/10.3390/dj7040111

29. Strobel-Schwarthoff K, Hirschfelder U, Hofmann E. Individualized erlanger KS-impression trays for infants with cleft lip and palate. Cleft Palate Craniofac J 2012;49:237-9. https://doi.org/10.1597/10103

How to cite this article: Kalaskar R, Bhaje P, Balasubramanian $S$, Kalaskar A. Effectiveness of the novel impression tray "cleftray" for infants with cleft lip and palate: a randomized controlled clinical trial. J Korean Assoc Oral Maxillofac Surg 2021;47:82-90. https://doi.org/10.5125/jkaoms.2021.47.2.82 